\title{
SUR LE SUCRE VIRTUEL DU SANG APRÈS LA LIGATURE DES VAISSEAUX RÉNAUX DOUBLES
}

\author{
Par \\ SACHIKADO MORITA. \\ （森出幸門） \\ (Du laboratoire de physiologie du Prof. Y. Satake de la \\ Tôhôku Lniversité impériale de Sendai.)

\section{I.-INTRODUCTION}

La chimie biologique moderne nous fait supposer que plusieures formes d'hydrates de carbone se rencontrent dans le sang.

A ce point de vue, F. W. Pav $y^{1)}$ avait établi cu'un extrait du saing de lapin devient plus réducteur si on le fait bouillir avec l'acide chlorhydrique. Il pensait que ce qui donnait une augmentation sensible du chiffre de la réduction après l'hydrolyse était un certain nombre des sucres ainsi que le glycose, et tous les sucres sont combinés à l'albumine dans le sang.

R. Lépin et ses collaborateurs ont distingué deux espèces des sucres du sang: $1^{\circ}$ le sucre immédict ou le sucre libre, que l'on obtient immédiatement par le simple dosage du sang et il correspond $\dot{a}$ ce qu'on appelle habituellement le sucre du sang, $2^{\circ}$ le sucre virtuel, celui qui exige, pour que la combinaison soit détruite, l'emploi de moyens énergiques, c'est à dire n'est dégagé que grâce à l'action d'invertine ou en étant chauffé avec l'acide. En outre, la proportion du sucre virtuel pour $100 \mathrm{du}$ sucre libre est appelé par eux sncre combiné. Quant à la provenance du sucre virtuel, Lép in e et d'autre ${ }^{3 / 3}$ sontiennent qu'une

1) F. W. Pav y, Physiology of carbohydrate, London 1894.

2) R. L ép ine, Diabète sucré, Paris 1909,57 ;-Le Sucre du sang, Paris 1!2:1, 9.

3) R. Lépine et R. B oulud, Journal de physiol. et pathol. génér., 1911, 13, 17 . 
certain portion de celui-ci a une origine exogène et l'autre portion endogène, car après l'ingestion $d u$ sucre on observe l'augmentation du sucre virtuel, notamment dans le sang de la veine porte, et chez des chiens à l'inanition depuis plusieurs jours on constate l'augmentation du sucre virtuel par des saignées. Donc Lépine a remarqué, que dans le cas endogène on ne voit guère d'autre sources du sucre virtuel que du glycogène, de l'acide glycuronique ou de sucre engagé en combinaison à l'albumine.

En 1912, H. Bierry et L. Fandard ${ }^{1)}$ ont publiè une méthode qui permit de doser non seulement le sucre libre, mais encore le sucre total du sang. Ils ont dosé le sucre libre et le sucre engagé en combinaison du sang artériel du chien, des oiseaux et du cheval, et ont fait l'interresante découverte que le taux du sucre engagé en combinaison varie suivant l'animal, et cela de façon inverse de celui du sucre libre. Par exemple, le sang des oiseaux, qui est le plus riche en sucre libre, renferme la plus faible proportion de sucre engagé en combinaison, et le sang du cheval, relativement pauvre en sucre libre, renferme au contraire une proportion relativement plus grande de sucre engagé en combinaison. Ainsi le taux du sucre total est constamment le même. Bierr $\mathrm{y}^{2}$ appele le sucre engagé en combinaison le sucre protéidique, parce que c'est celui qu'est dégagé des substances protéiques que l'on trouve en extrayant le sang par l'hydrolyse avec l'acide.

Si le sucre virtuel était ce qu'est présumé par Lépine, on derrait avec raison s'attendre à des variations de sa quantité à la suite de troubles apportés dans l'état pathologique.

Dáprès mes expériences faites depuis plusieurs années sur des lapins en pratiquant la ligature des vaisseaux rénaux doubles, le sucre du saug, comme on générallement dit, dosagé par la méthode microchimique de Bang, recoit une angmentation si remarquable, en outre de l'hyperglycémie temporaire provoqèe par l'opération elle-même, quelques jours avant le mord d'animals, et la section des splanchniques ne peut pas arrête l'aparition de cette hyperglycémie."

J'ai fait dans le present travail nombre d'expériences en ligaturant les vaisseaux rénaux doubles chez les lapins, et ai comparé les chiffres du sucre combiné avant et après la ligature dans la période agonique.

1) H. Bierry et L. Fandard, C. R. de la Société de Biologie, 1912, 72, 928

2) H. Bierry et A. Ranc, C. K. de l'Acad. des Sciences, 1914, 158, 278.

3) Sachi. Morita, Tohoku Journ. of Exp. Med., 1922, 3, 226. 


\section{II.-MÉrHODES.}

Animaux: lapins mâles et sains, nourris de tofukara (residu des pois soja) depuis au moins dix à quinze jours.

Ligature des vaisseux rénaux doubles: par la voie dorsolornbaire. Après la coupe des poils sur le flanc, la peau est lavée et désinfectée au moyen de la teinture de iode. On fait sur la peau une incision longitudinale d'uno étendue de 4 centimètres, à une distance de 1-3 centimètres en arrière du dernier côte de droite et de gauche, et à 2-3 centimètres de la vertèbre. De cette manière on a coupé la peau et le tissue souscutané jusqu'a ce que on tombe sur l'aponéverose dorsolombaire. Au niveau du bord inférieur du grand dorsal et du bord supérieur de l'oblique externe de l'abdomen, on pénètre dans l'abdomen en enfoncant une pince. L'ouverture êtant suffisante, on introduit un fil de soie stérilisée avec la sonde sous les vaisseaux avec précaution pour ne pas perforer le péritoine. Ensuite on met une solide ligature sur vaisseaux artériel et veineux en bloc. La fermeture de l'ouverture s'opère à l'aide d'une double suture, comprenant d'abord les parties musculaires et ensuite l'incision des téguments.

Echantillons de sang: le sang du rameau postérieur de la veine auriculaire était dosagé par la méthode microchimique de I. Bang ${ }^{12}$. - Pour le dosage du sucre libre et virtuel on emploiait la méthode de R. Lépine et R. Boulud ${ }^{2 ; 3)}$. Dans ce cas on emploie une bouteille en plomb d'un demi-litre surmontée d'un assez long tube en plomb soudé au col de la bouteille et entouré d'un réfrigérant ascendant.

\section{III.-EFFETS DE L'INANITION SUR LE SUCRE VIRTUEL.}

Après la ligature des vaisseaux rénaux doubles, l'animal commence à être malade et veut ordinairement ni boire, ni manger, ou bien très rarement et toujours en petite quantité jusqu'à sa mort. Je ne lui donnait donc pas d'aliment pendant l'expérience. Et j'ai fait ainsi plusieurs expériences pour controler l'effets de l'inanition sur le sucre combiné du sang.

I) I. B ang, Bioch. Ztschr., 1913, 49, 19 et 1913, 57, 300 .

2) R. Boulud, Journal de physiol. et pathol. génér., 1909, 11, 12.

3) R. Lép ine et R. Boulu d, Journal de physiol. et pathol. génér., 1911, 13, 178. 
Cl. Bernar $d^{1)}$ a remarqué que l'inanition semble augmenter un peu la quantité du sucre dans le sang pendant la première période, mais diminuer enfin dans la période agonique. D'après v. Mering ${ }^{2)}$ et J. G. Otto $0^{3)}$, le sucre du sang pendant l'inanition durant plusiers jours est ni augmenté ni diminué. A. Imamura et G. Kira ${ }^{4}$ observaient la même chose, mais au moment de la mort, ils constataient que les quantités de matière sucrées sont très faibles. J'ai ${ }^{52}$ trouvé aussi précédement que le sucre du sang pendant l'inanition semble augmenter plus ou moins, mais ne subit pas de variations importantes jusqu'à la période agonique où il diminue peu a peu.

H. Bierry et L. Fandard ${ }^{6)}$ ont déterminé le sucre libre et le sucre protéidique pendant l'inanition et ont remarqué que le premier reste presque constant dans la première période (du première ou douzième

TABLEAU

\begin{tabular}{|c|c|c|c|c|}
\hline $\begin{array}{c}\text { Numéro } \\
\text { de } \\
\text { l'expérience }\end{array}$ & Date & $\begin{array}{l}\text { Poids du } \\
\text { corps }\end{array}$ & Temps & $\begin{array}{l}\text { Température } \\
\text { du corps }\end{array}$ \\
\hline Lapin 1. & $\begin{array}{l}\text { 2. XI. } 1921 \\
\text { 5. XI. " }\end{array}$ & $\begin{array}{l}1^{\mathrm{kgr}}, 73 \\
1^{\mathrm{kgr}}, 55\end{array}$ & $\begin{array}{c}10 \text { h. } 00 \text { A.M. } \\
9 \text { h. } 20 "\end{array}$ & $\begin{array}{l}39^{\circ}, 1 \\
37^{\circ}, 9\end{array}$ \\
\hline Lapin 2. & $\begin{array}{l}\text { 9. XI. } 1921 \\
\text { 13. XI. " }\end{array}$ & $\begin{array}{l}2^{\mathrm{kgr}}, 42 \\
2^{\mathrm{kgr}}, 10\end{array}$ & $\begin{array}{l}10 \text { h. } 00 \text { A.sr. } \\
10 \text { h. } 00 "\end{array}$ & $\begin{array}{l}39^{\circ}, 3 \\
38^{\circ}, 2\end{array}$ \\
\hline Lapin 3. & $\begin{array}{l}\text { 16. XI. } 1921 \\
\text { 20. XI. " }\end{array}$ & $\begin{array}{l}1^{\mathrm{kgr}}, 95 \\
1^{\mathrm{kgr}}, 58\end{array}$ & $\begin{array}{l}10 \text { h. } 00 \text { A.M. } \\
10 \text { h. } 00 "\end{array}$ & $\begin{array}{l}38^{\circ}, 9 \\
37^{\circ}, 5\end{array}$ \\
\hline Lapin 4. & $\begin{array}{l}\text { 23. XI. } 1921 \\
25 . \text { XI. " }\end{array}$ & $\begin{array}{l}1^{\mathrm{kgr}}, 61 \\
1^{\mathrm{kgr}}, 48\end{array}$ & $\begin{array}{l}10 \text { h. } 00 \text { A.Mr. } \\
10 \text { h. } 00 \text { " }\end{array}$ & $\begin{array}{l}38^{\circ}, 4 \\
38^{\circ}, 4\end{array}$ \\
\hline Lapin 5. & $\begin{array}{l}\text { 28. XI. } 1921 \\
\text { 1. XII. " }\end{array}$ & $\begin{array}{l}2^{\mathrm{kgr}}, 06 \\
1^{\mathrm{kgr}}, 84\end{array}$ & $\begin{array}{l}10 \text { h. } 00 \text { A.M. } \\
2 \text { h. } 00 \text { P.M. }\end{array}$ & $\begin{array}{l}38^{\circ}, 0 \\
38^{\circ}, 2\end{array}$ \\
\hline Lapin 6. & $\begin{array}{l}\text { 30. XI. } 1921 \\
\text { 3. XII. " }\end{array}$ & $\begin{array}{l}2^{\mathrm{kgr}}, 12 \\
1^{\mathrm{kgr}}, 88\end{array}$ & $\begin{array}{l}10 \text { h. } 00 \text { A.M. } \\
12 \text { h. } 00 \text { м. }\end{array}$ & $\begin{array}{l}37^{\circ}, 9 \\
37^{\circ}, 7\end{array}$ \\
\hline Lapin 7. & $\begin{array}{l}\text { 7. XII. } 1921 \\
\text { 10. XII. " }\end{array}$ & $\begin{array}{l}1^{\mathrm{kgr}}, 80 \\
1^{\mathrm{kgr}}, 62\end{array}$ & $\begin{array}{l}9 \text { h. } 30 \text { A.M. } \\
10 \text { h. } 30 \text { " }\end{array}$ & $\begin{array}{l}38^{\circ}, 4 \\
37^{\circ}, 8\end{array}$ \\
\hline Lapin 8. & $\begin{array}{l}\text { 13. XII. } 1921 \\
\text { 16. XII. " }\end{array}$ & $\begin{array}{l}2^{\mathrm{kgr}}, 10 \\
1^{\mathrm{kgr}}, 80\end{array}$ & $\begin{array}{l}9 \text { h. } 00 \text { A.m. } \\
9 \text { h. } 30 " n\end{array}$ & $\begin{array}{l}38^{\circ}, 6 \\
37^{\circ}, 7\end{array}$ \\
\hline
\end{tabular}

1) Cl. Bernard, Leçons sur le diabète, Paris, 1877, 210.

2) r. Mering, Arch. f. Physiol., 1877, 388.

3) J. G. Ot to, Pflüg er's Arch., 18\$5, 35, 492. 
jour), et diminue dans la période agonique qui dure quatre ou cinq jours, tandis que le dernier augmente généralement dans la seconde période, et surtout dans la période agonique où il arrive à son maximum.

Le matin, deux échantillons du sang sont prélevé sur chaque animal, à jeun, immédiatement a vant le début de l'expérience. L'un pris de la veine auriculaire est destiné pour dosage du sucre du sang générallement dit, et l'autre pris de la carotide pour le dosage du sucre libre et virtuel Ces animaux sont ensuite mis à l'inanition, on ne leur donna pas même de l'eau. Après l'inanition, qui durait environ de cinquante au cent heures, on a répété des saignées pour le dosage de sucre libre et virtuel.

Voici les résultats.

I.

\begin{tabular}{|c|c|c|c|c|c|}
\hline \multirow{2}{*}{$\begin{array}{l}\text { Durée de } \\
\text { la diête } \\
\text { en heur }\end{array}$} & \multirow{2}{*}{$\begin{array}{l}\text { Sucre pour } 100 \\
\text { gr. du sang } \\
\text { d'après la mé- } \\
\text { thode microchi- } \\
\text { mique de } \\
\text { Bang }\end{array}$} & \multicolumn{3}{|c|}{$\begin{array}{l}\text { Sucres pour } 1000 \mathrm{gr} \text {. du sang d'après la } \\
\text { méthode de Lép ine }\end{array}$} & \multirow{2}{*}{$\begin{array}{c}\text { Sucre } \\
\text { combiné p. } \\
100 \text { du sucre } \\
\text { libre }\end{array}$} \\
\hline & & Sucre libre & Sucre virtuel & Sucre total & \\
\hline 71 & $\begin{array}{l}0 \mathrm{gr}, 11 \\
0 \mathrm{gr}, 12\end{array}$ & $\begin{array}{l}1 \mathrm{gr}, 170 \\
1 \mathrm{gr}, 295\end{array}$ & $\begin{array}{l}1 \mathrm{gr}, 078 \\
0 \mathrm{gr}, 903\end{array}$ & $\begin{array}{l}2 g r, 248 \\
2 g r, 198\end{array}$ & $\begin{array}{l}92 \\
70\end{array}$ \\
\hline 96 & $\begin{array}{l}0 \mathrm{gr}, 10 \\
0 \mathrm{gr}, 10\end{array}$ & $\begin{array}{l}1 \mathrm{gr}, 215 \\
1 \mathrm{gr}, 555\end{array}$ & $\begin{array}{l}1 \mathrm{gr}, 425 \\
0 \mathrm{or}, 995\end{array}$ & $\begin{array}{l}22 \mathrm{r}, 640 \\
2 g \mathbf{r}, 550\end{array}$ & $\begin{array}{r}117 \\
64\end{array}$ \\
\hline 100 & $\begin{array}{l}0 \mathrm{gr}, 10 \\
0 \mathrm{gr}, 09\end{array}$ & $\begin{array}{l}1 \mathrm{gr}, 045 \\
1 \mathrm{gr}, 500\end{array}$ & $\begin{array}{l}0 \mathrm{gr}, 910 \\
1 \not \mathrm{rr}, 090\end{array}$ & 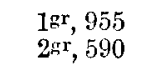 & $\begin{array}{l}87 \\
73\end{array}$ \\
\hline 48 & $\begin{array}{l}0 \mathrm{gr}, 08 \\
0 \mathrm{gr}, 11\end{array}$ & $\begin{array}{l}\operatorname{lgr}, 485 \\
1 \mathrm{gr}, 530\end{array}$ & $\begin{array}{l}1 \mathrm{gr}, 215 \\
1^{\mathrm{gr}}, 060\end{array}$ & $\begin{array}{l}2 \stackrel{\circ}{a r}, 700 \\
2 \mathrm{gr}, 590\end{array}$ & $\begin{array}{l}82 \\
69\end{array}$ \\
\hline 76 & $\begin{array}{l}0 \mathrm{gr}, 08 \\
0 \mathrm{sr}, 08\end{array}$ & $\begin{array}{l}1 \mathrm{gr}, 350 \\
1 \mathrm{gr}, 265\end{array}$ & $\begin{array}{l}0 \mathrm{gr} \mathbf{r}, 710 \\
0 \mathrm{gr}, 613\end{array}$ & $\begin{array}{l}2 \underset{r}{r}, 060 \\
1 \mathbf{r r}, 878\end{array}$ & $\begin{array}{l}53 \\
48\end{array}$ \\
\hline $7 t$ & $\begin{array}{l}0 \mathrm{gr}, 09 \\
0^{\mathrm{gr}}, 08\end{array}$ & $\begin{array}{l}1 \mathrm{gr}, 240 \\
1 \mathrm{gr}, 528\end{array}$ & 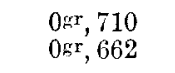 & $\begin{array}{l}1 \mathrm{rr}, 950 \\
2 \mu \mathrm{r}, 190\end{array}$ & $\begin{array}{l}57 \\
43\end{array}$ \\
\hline 73 & $\begin{array}{l}0 \mathrm{gr}, 10 \\
0 \mathrm{gr}, 09\end{array}$ & $\begin{array}{l}1 \mathrm{gr}, 265 \\
1 \mathrm{gr}, 715\end{array}$ & $\begin{array}{l}0 \backsim \mathrm{r}, 745 \\
0 \mathrm{ar}, 695\end{array}$ & $\begin{array}{l}2 \mathrm{r}, 010 \\
2 \mathrm{rr}, 410\end{array}$ & $\begin{array}{l}59 \\
40\end{array}$ \\
\hline 72 & $\begin{array}{l}0 \mathrm{gr}, 09 \\
0 \mathrm{gr}, 11\end{array}$ & $\begin{array}{l}1 \mathrm{gr}, 755 \\
1 \mathrm{gr}, 470\end{array}$ & $\begin{array}{l}1 \mathrm{gx}, 025 \\
1^{\mathrm{gr}}, 020\end{array}$ & $\begin{array}{l}2 \mathrm{gr}, 780 \\
2 \mathrm{rr}, 490\end{array}$ & $\begin{array}{l}58 \\
70\end{array}$ \\
\hline
\end{tabular}

4) Imamura et G. K ira, Nippon-Naikwagakwai-Zasshi, J920, 7, 676 (jap.).

5) Sachi. Morit a, Tohoku Journ. of Exper. Med., 1922, 3, 226.

6) H. Bierry et L. Fandard, C. R. de l'Acad. des Sciences, 1919, 156, 2010. 
Les chiffres du sucre du sang par la méthode microchimique ne semblent pas subir de variations remarquable comme je l'ai dit précédemment.

Le sucre libre semble un peu augmenter. Le taux du sucre libre avant l'expérience est de 1 gr. $045-1$ gr. 755 , en moyenne 1 gr. 315, pour 1000 gr. de sang, et celui-ci d'après l'expérience est de 1 gr. 265-1 gr. 715, en moyenne 1 gr. 482.

Le sucre virtuel avant l'expérience est 0 gr. $710-1$ gr. 425 , en moyenne 0 gr. 977 ; et celui après 1'inanition est 0 gr. $613-1$ gr. 090 , en moyenne 0 gr. 879.

Quant au sucre combiné, il y a diminution sensible après l'inanition, mais elle ne se monte jamais à 50 pour 100 de la quantité étant trouvée avant l'inanition. C'est à dire; avant l'inanition 53-117, en moyenne 76 , et après l'inanition $40-73$, en moyenne 59 .

\section{V.-Effets de la ligature des varsseaux REnaux doubles} SUR LE SUCRE VIRTUEL DU SANG.

J'ai remarqué précédemment que le sucre du sang, dosagé par la méthode de $\mathrm{Bang}$, après la ligature des vaisseaux rénaux doubles chez le lapin, augmente après disparaitre de l'hyperglycémie temporaire par cette opération, de plus en plus des le lendemain jusqu'à sa mort, et que cette augmentation n'est pas de nature centrale, parce qu'elle ne disparait pas après la coupe des nerfs splanchniques doubles.

Dans l'expériences suivantes, j'ai fait d'abord des saignées pour le dosage du sucre du sang normal. Ensuite on fait la ligature des vaisseaux rénaux par la voie dorso-lombaire de l'animal. Je ne lui ai pas donné, comme je l'ai dit précédemment, ni à boire ni à manger. Après cinquante au soixante-dix heures environ, lorsque les phénomènes urémiques se sont décairés, j’ai fait des saignées pour la deuxième fois.

Voici les protocoles de quelques-unes de ces expériences. 
EXPÉRIFATCE III.

\begin{tabular}{|c|c|c|c|c|c|c|c|c|c|}
\hline \multirow[b]{2}{*}{ 亗 } & \multirow{2}{*}{ 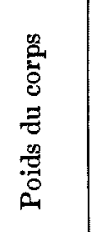 } & \multirow[b]{2}{*}{$\stackrel{2}{\stackrel{2}{3}}$} & \multirow{2}{*}{ 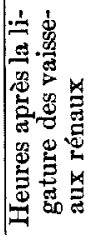 } & \multirow{2}{*}{ 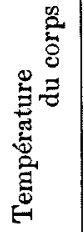 } & \multirow{2}{*}{ 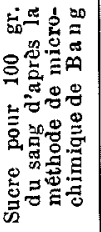 } & \multicolumn{3}{|c|}{$\begin{array}{l}\text { Sucre pour } 1000 \mathrm{gr} \text {. du } \\
\text { sang d'après la méthode } \\
\text { de L épine }\end{array}$} & \multirow{2}{*}{ 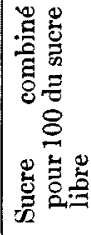 } \\
\hline & & & & & & 总 & 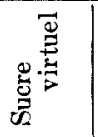 & 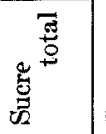 & \\
\hline 17. I.'22 & $1^{\mathrm{kgr}, 65}$ & $\begin{array}{c}\text { A.M. } \\
10 \text { h. } 00 \\
10 \text { h. } 30- \\
10 \text { h. } 48 \\
\text { P M. } \\
8 \text { h. } 00\end{array}$ & & $\begin{array}{r}38^{\circ}, 8 \\
\text { Liga } \\
38^{\circ}, 2\end{array}$ & $\begin{array}{c}0^{\mathrm{gr}}, 11 \\
\text { ure des } \\
0^{\mathrm{gr}}, 11\end{array}$ & $\begin{array}{r}1 \mathrm{gr}, 075 \\
\text { isseaux } r\end{array}$ & $\begin{array}{l}\text { ogr }, 930 \\
\text { naux }\end{array}$ & $2^{\mathrm{gr}}, 005$ & 87 \\
\hline 18. I. " & $1^{\mathrm{kgr}, 45}$ & $\begin{array}{c}\text { A.M. } \\
9 \text { h. } 00 \\
\text { P.M. } \\
8 \text { h. } 00\end{array}$ & & $\begin{array}{l}37^{\circ}, 2 \\
37^{\circ}, 8\end{array}$ & $\begin{array}{l}0 \mathrm{gr}, 11 \\
0^{\mathrm{gr}}, 11\end{array}$ & & & & \\
\hline 19. I. " & $1^{\mathrm{kgr}, 41}$ & $\begin{array}{c}\text { A.M. } \\
9 \text { h. } 00 \\
\text { P.M. } \\
3 \text { h. } 30\end{array}$ & 53 & $\begin{array}{l}35^{\circ}, 0 \\
35^{\circ}, 4\end{array}$ & $\begin{array}{l}0 \mathrm{gr}, 19 \\
0^{\mathrm{gr}}, 30\end{array}$ & $3 \mathrm{gr}, 695$ & $1 \mathrm{gr}, 337$ & $5^{\mathrm{gr}}, 032$ & 36 \\
\hline
\end{tabular}

Expérience V.

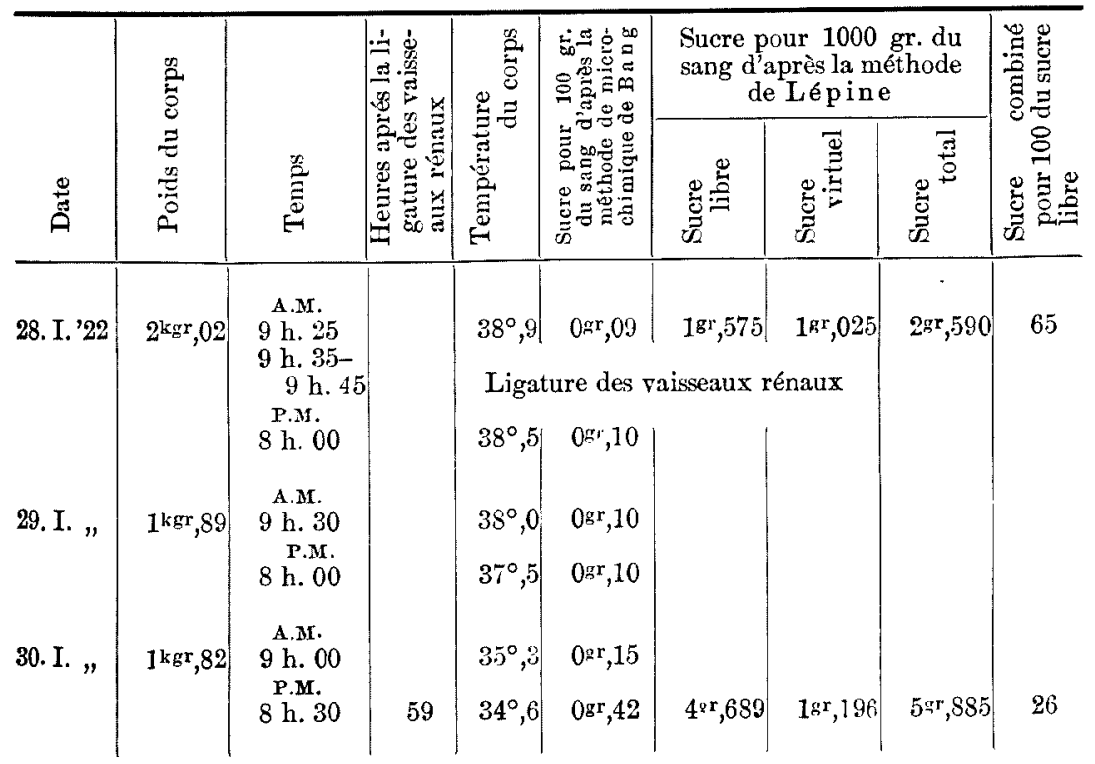


ExṔ́RIENCE VI.

\begin{tabular}{|c|c|c|c|c|c|c|c|c|c|}
\hline \multirow[b]{2}{*}{$\stackrel{\mathscr{\tilde { B }}}{\tilde{\theta}}$} & \multirow{2}{*}{ 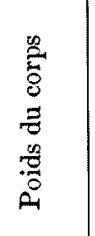 } & \multirow[b]{2}{*}{ 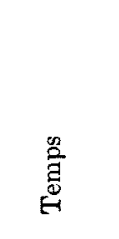 } & \multirow{2}{*}{ 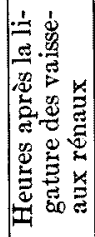 } & \multirow{2}{*}{ 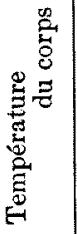 } & \multirow{2}{*}{ 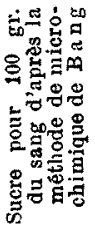 } & \multicolumn{3}{|c|}{$\begin{array}{c}\text { Sucre pour } 1000 \mathrm{gr} \text {. du } \\
\text { sang d'après la méthode } \\
\text { de Lépine }\end{array}$} & \multirow{2}{*}{ 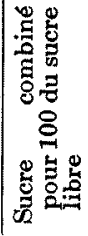 } \\
\hline & & & & & & 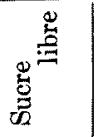 & 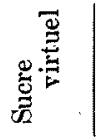 & 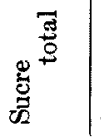 & \\
\hline 2. II. ' 22 & $1^{\mathrm{kgr}, 95}$ & $\begin{array}{c}\text { A.M. } \\
9 \text { h. } 00 \\
9 \text { h. } 30- \\
9 \text { h. } 40 \\
\text { P.M. } \\
5 \text { h. } 00\end{array}$ & & $\begin{array}{r}38^{\circ}, 4 \\
\text { Ligat } \\
38^{\circ}, 4\end{array}$ & $\begin{array}{r}0 \mathrm{gr}, 10 \\
\text { ure des } \\
0^{\mathrm{gr}}, 14\end{array}$ & $\begin{array}{r}1 \mathrm{gr}, 095 \\
\text { isseaux } \mathrm{r}\end{array}$ & $\begin{array}{c}0 \mathrm{gr}, 981 \\
\text { naux }\end{array}$ & $2 \mathrm{gr}, 076$ & 90 \\
\hline 3. II. , & $1^{\mathrm{kar}, \mathrm{s0}}$ & $\begin{array}{l}\text { A.M. } \\
9 \text { h. } 00 \\
\text { P.M. } \\
9 \text { h. } 00\end{array}$ & & $\begin{array}{l}37^{\circ}, 6 \\
38^{\circ}, 2\end{array}$ & $\begin{array}{l}0 \mathrm{gr}, 12 \\
0^{\mathrm{gr}}, 14\end{array}$ & & & & \\
\hline 4. II. " & $1 \mathrm{kgr}, 75$ & $\begin{array}{l}\text { A.M. } \\
8 \text { h. } 30 \\
\text { P.M. } \\
4 \text { h. } 30\end{array}$ & 55 & $\begin{array}{l}36^{\circ}, 6 \\
37^{\circ}, 6\end{array}$ & $\begin{array}{l}0 \mathrm{gr}, 19 \\
0 \mathrm{gr}, 16\end{array}$ & $1 \mathrm{gr}, 715$ & $1 \mathrm{gr}, 148$ & $2 \mathrm{gr}, 863$ & 66 \\
\hline
\end{tabular}

Tableav II.

\begin{tabular}{|c|c|c|c|c|c|c|c|}
\hline \multirow{2}{*}{$\begin{array}{l}\text { Numero } \\
\text { de } \\
\text { l'expér- } \\
\text { ience }\end{array}$} & \multicolumn{3}{|c|}{ Avant la ligature } & \multirow{2}{*}{$\begin{array}{c}\text { Heures } \\
\text { de la } \\
\text { ligature } \\
\text { au } \\
\text { dosage } \\
\text { second }\end{array}$} & \multicolumn{3}{|c|}{ Après la ligature } \\
\hline & $\begin{array}{l}\text { Sucre } \\
\text { libre }\end{array}$ & $\begin{array}{l}\text { Sucre } \\
\text { virtuel }\end{array}$ & $\begin{array}{c}\text { Sucre } \\
\text { combiné }\end{array}$ & & $\begin{array}{c}\text { Sucre } \\
\text { libre }\end{array}$ & $\begin{array}{l}\text { Sucre } \\
\text { virtuel }\end{array}$ & $\begin{array}{l}\text { Sucre } \\
\text { combiné }\end{array}$ \\
\hline $1^{\circ}$ (21. XII.'21) & $1 \mathrm{gr}, 145$ & $0 \mathrm{gr}, 865$ & 77 & 53 & $6 \mathrm{gr}, 120$ & $1^{\mathrm{gr}}, 046$ & 17 \\
\hline $2^{\circ}(7 . \quad$ I. 22$)$ & $1 \mathrm{gr}, 250$ & $0 \mathrm{gr}, 608$ & 49 & 71 & $2^{g r}, 521$ & $1{ }^{\mathrm{gr}}, 009$ & 40 \\
\hline $3^{\circ}(17 . \quad$ I. , ) & $18 \mathrm{gr}, 075$ & $0 \mathrm{gr}, 930$ & 86 & 53 & $3^{\mathrm{gr}}, 695$ & $1^{\mathrm{gr}}, 339$ & 36 \\
\hline $4^{\circ}(23$ & $1 \mathrm{gr}, 005$ & $0 \mathrm{gr}, 935$ & 93 & 53 & $2 \mathrm{gx}, 7 \mathrm{~S} 4$ & $0^{\mathrm{gr}}, 932$ & 33 \\
\hline $5^{\circ}(28 . \quad$ I. , ) & $1^{g x}, 575$ & $1^{\mathrm{gr}, 025}$ & 65 & 59 & $4 \mathrm{gr}, 689$ & $1^{\mathrm{gr}}, 196$ & 25 \\
\hline $6^{\circ}(2, \quad$ II. , $)$ & $1 \mathrm{gr}, 095$ & $0 \mathrm{gr}, 981$ & 90 & 55 & $1 \mathrm{gr}, 715$ & $1^{\mathrm{gr}}, 148$ & 67 \\
\hline $7^{\circ}(7 . \quad$ II.,$)$ & $1 \mathrm{gr}, 570$ & $0^{\mathrm{kr},}, 920$ & 59 & 58 & $4^{\approx r}, 477$ & $1^{\mathrm{gr}}, 228$ & 27 \\
\hline
\end{tabular}

De ce tableau on voit bien que le sucre du sang, dosage par la méthode de Bang, augmente si fort après la ligature des vaisseaux rénallx, que j'ai trouvé dans une première série d'expériences. C'est à dire, avant la ligature 0 gr. $09-0$ gr. 13 , en moyen ne 0 gr. 105 et après 
la ligature $0 \mathrm{gr} .16-0$ gr. 56 , en moyenne 0 gr. 33 pour $100 \mathrm{gr}$. de sang.

Le sucre libre a augmenté remarquablement. Chez six lapins (excepté No. 6) le taux avant la ligature est $1 \mathrm{gr} .005-1 \mathrm{gr} .575$ (en moyenne $1 \mathrm{gr} .270$ ), pour $1000 \mathrm{gr}$. du sang. Et celui-ci 50-90 heures après la ligature est $1 \mathrm{gr} .715-6$ gr. 120 (en moyenne $3 \mathrm{gr} .869$ ). Le sucre virtuel avant 0 gr. $608-1$ gr. 025 (en moyenne 0 gr. 830) avant la ligature et 0 gr. $932-1 \mathrm{gr} .339$ (en moyenne $1 \mathrm{gr}$. 125) 50-90 heures après l'operation.

Expérience VI doit être une exclude, parce que l'hyperglycémie du derniers jours non était pas encore grande.

Soir le sucre libre et le sucre total du sang augmentent aussi parallèlement, par conséquent, le sucre virtuel n'a pas subi de variation importantes, mais il augmente plus on moins après la ligature.

Quant au sucre combiné, j'ai trouvé toujours la diminution très forte. Tandis que la diminution du sucre combiné à l'animal, mis à l'inanition, ne se monte pas à 50 pour 100 du chiffre avant l'inanition ; celle de l'animal dont les vaisseaux rénaux sont ligaturé depuis plusieurs jours est toujours 50 pour 100 du chiffre avant la ligature.

\section{V.-Conclusrons. *}

Le sucre virtuel du sang augmentait plus ou moins après la ligature des vaisseaux rénaux doubles.

Le sucre combiné du sang après la ligature diminue, parce que l'augmentation du sucre libre était plus forte que celle du sucre virtuel.

* N.B. à l'occasion de la correction: H. Bierry, F. Rathery, et F. Bordat récement rapportaient l'élévation du taux du sucre protéidique dans la plasma sanguin par ligature des deux urétères chez le chien, mais ils n'observaient pas une remarquable augmentation du sucre libre pendant 2 ou 3 jours après ligature, au contraire des résultats de més expériences chez le lapin [C. R. de l'Acad. des Scien. 174, No.14 (3 April 1922) 970]. 